\title{
22 UNINTENTIONAL INJURY MORTALITY IN INDIA, 2005: NATIONALLY REPRESENTATIVE MORTALITY SURVEY OF 1.1 MILLION HOMES
}

doi:10.1136/injuryprev-2012-040580b.22

\footnotetext{
${ }^{1,2} \mathrm{~J}$ Jagnoor*, ${ }^{2} \mathrm{~W}$ Suraweera, ${ }^{1} \mathrm{~L}$ Keay, ${ }^{1} \mathrm{R}$ Ivers, ${ }^{3} \mathrm{~J}, \mathrm{~S}$ Thakur, ${ }^{2} \mathrm{P}$ Jha,for the Million Death Study Collaborators. ${ }^{1}$ The George Institute for Global Health and Sydney School of Public Health, The University of Sydney, Sydney, Australia; ${ }^{2}$ Centre for Global Health Research, Li Ka Shing Knowledge Institute, St. Michael's Hospital, and Dalla Lana School of Public Health, University of Toronto, Toronto, Canada; ${ }^{3}$ School of Public Health, Post Graduate Institute of Medical Education and Research, Chandigarh, India
}

Background Unintentional injuries are an important cause of death in India. However, no reliable nationally representative estimates of unintentional injury deaths are available.

Aim To estimate total unintentional injury mortality in India using results from a nationally representative survey of the causes of deaths.

Methods Unintentional injury deaths were examined in a nationally representative mortality survey covering 123000 deaths occurring in 1.1 million homes. Trained non-medical field staff to interview all households in which a death had occurred from 2001 to 2003 within its Sample Registration System. Structured field reports detailing the events preceding death were obtained from living relatives of the deceased and emailed to two of 130 trained physicians who independently assigned an underlying cause to each death. Cause specific mortality proportions were applied to all-cause mortality estimates from the United Nations for the year 2005.

Results In the year 2005, unintentional injury caused 648000 deaths (7\% of all deaths; 58/100 000 population). Mortality rates were higher among males than females, and in rural versus urban areas. Road traffic injuries (185000 deaths; 29\% of unintentional injury deaths), falls (160 000 deaths, 25\%) and drowning (73 000 deaths, $11 \%$ ) were the three leading causes of unintentional injury mortality. The highest unintentional mortality rates were in those aged 70 years or older $(410 / 100000)$ mostly due to falls. 